UVX 2010 (2011) 41-47

DOI: $10.1051 / \mathrm{uvx} / 2011006$

(C) Owned by the authors, published by EDP Sciences, 2011

\title{
SAMBA: The 4-40 keV X-ray absorption spectroscopy beamline at SOLEIL
}

\author{
V. Briois, E. Fonda, S. Belin, L. Barthe, C. La Fontaine, F. Langlois, \\ M. Ribbens and F. Villain
}

Synchrotron SOLEIL, L'Orme des Merisiers, BP. 48, 91191 Gif-sur-Yvette, France

\begin{abstract}
The SAMBA X-ray Absorption beamline has been operating for users since January 2008. The beamline covers a large energy range from 4 up to $40 \mathrm{keV}$ in two working modes: a "high flux" mode using a sagittally focusing monochromator and a "time-resolved" mode using a dedicated Quick-EXAFS monochromator. Herein a technical description of the main beamline components is given and results concerning energy resolution, available flux and time resolved performances are presented.
\end{abstract}

\section{Introduction}

Today X-ray absorption spectroscopy (XAS) is a versatile and well established technique for providing electronic and structural information on all kind of materials. As the technique is widely reviewed by specialized books and articles [1-4], only a brief review of XAS is given here. Its advent was strongly associated with the development of unique X-rays sources which are the Synchrotron Radiation (SR) facilities. The technique benefits from almost all the outstanding features of the SR, such as the continuous energy spectrum, the high flux, the natural collimation and linear polarization. The physical process underlying the XAS is the ejection of an electron, called photo-electron, when X-rays are absorbed by the matter. This phenomenon arises when incident X-rays energy is scanned across the range corresponding to that required for the ionization of a core electron from an atom, called absorbing atom, in the sample. The interference between the outgoing wave associated to the photo-electron and the waves originating from the backscattering of this photo-electron by the neighbouring atoms of the absorbing atom gives rise to oscillatory structures on the X-ray absorption spectrum, arbitrarily divided into Extended X-ray Absorption Fine Structures (EXAFS) and X-ray Absorption Near Edge Structures (XANES). On one hand, EXAFS is one of the few structural techniques that provide information over the short-range order (inter-atomic distances, types and number of first and more distant neighbours) around almost any atomic species of the periodic table. On the other hand, complementary information are obtained from the analysis of the XANES spectrum, like the valence state, spin state and site symmetry of the selected absorbing atom. In contrast to X-ray diffraction, XAS is an element-specific probe, which can be applied to materials without long-range crystalline order. It allows the experimentalist to characterise the structural and electronic properties of a system, whatever the state of the sample, solid, liquid or gas and for atomic concentrations ranging from a few ppm to the pure element.

SOLEIL (Source Optimisée de Lumière d'Energie Intermédiaire de LURE) is a third generation synchrotron radiation source with a critical energy of $8.65 \mathrm{keV}$. The facility is composed of a $2.75 \mathrm{GeV}$ electron storage ring associated to a synchrotron booster. The stored current for user beamtime in multibunch filling mode is $400 \mathrm{~mA}$ with a top-up injection mode. The maximum stored current attained in the machine is $500 \mathrm{~mA}$ and should be the reference value for multi-bunch beamtime sessions in the near future. The facility has opened its first beamlines to users at the beginning of 2008. Among them, the SAMBA (Spectroscopies Applied to Materials Based on Absorption) beamline is dedicated

This is an Open Access article distributed under the terms of the Creative Commons Attribution-Noncommercial License 3.0, which permits unrestricted use, distribution, and reproduction in any noncommercial medium, provided the original work is properly cited. 
to XAS. SAMBA, which is one of the most over-subscribed SOLEIL's beamlines, is widely used by a large community of scientists and engineers in the fundamental and applied research fields, including Chemistry, Physics, Environmental Sciences, Biology and Surface Sciences. In the following are described the main beamline optical components and beamline facilities.

\section{Samba beamline description}

The SAMBA beamline, located on the bending magnet D09-1 is characterized by one lead-shielded enclosure containing the beamline optics and two experimental lead-shielded enclosures used for conventional XAS experiments and surface XAS investigation (SurfAS hutch), respectively.

\subsection{Optical layout}

The layout and main optical components located in the first lead-shielded radioprotection hutch are shown in Figure 1. The beamline is designed to be used from 4 to $40 \mathrm{keV}$ with two operation modes: a high flux mode using a dynamically sagittally focusing monochromator (d) and a time-resolved mode using a dedicated Quick-EXAFS monochromator (e). Both operation modes use the same optical components for defining the beam in the vertical plane, i.e. a collimating cylindrically bent mirror (b) and a focusing cylindrically bent mirror $(\mathrm{g})$ installed before and after the monochromators, respectively. The primary slits (a) are located at $12.8 \mathrm{~m}$ from the source, they define the horizontal and vertical dimensions of the polychromatic beam impinging the first mirror (b). X-ray sensitive screens (c, $f$ and h) can be inserted just after the optics as diagnostic tools. A monochromatic shutter (i) is installed at the end of the hutch for allowing the experimenter to access experimental hutches while keeping a constant heat load on the optics.

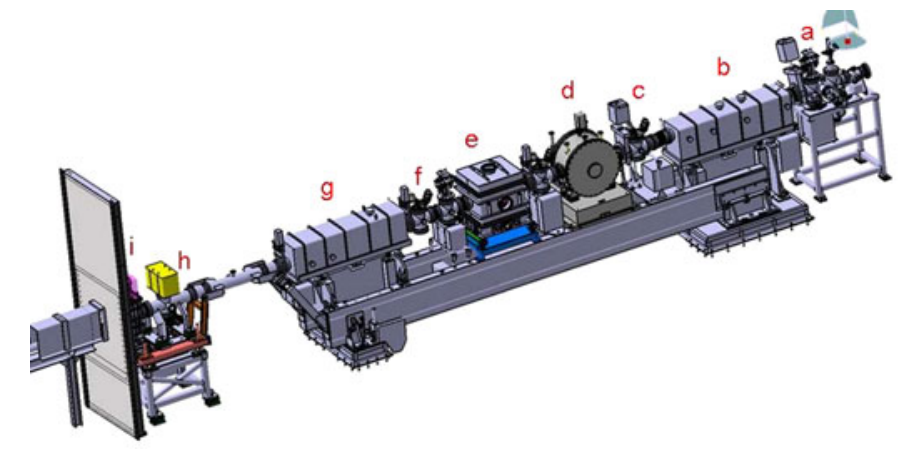

Figure 1. Optical layout of the SAMBA beamline.

\subsection{Collimating and focusing mirrors}

Two cylindrically bendable silicon mirrors coated by a layer of $50 \mathrm{~nm}$ of $\mathrm{Pd}$ are used for providing vertical i) collimation and ii) focusing of the beam at the sample position. The mirrors are used over the whole 4-40 keV energy range available on SAMBA by tilting the mirrors from $10 \mathrm{mrad}$ to $1 \mathrm{mrad}$ depending on the desired cut-off energy for removing the harmonic X-rays [5]. The reflective $\mathrm{Pd}$ surfaces are $1200 \mathrm{~mm}$ long per $88 \mathrm{~mm}$ wide allowing a horizontal acceptance of $\sim 6.2 \mathrm{mrad}$ on the first collimating mirror located at $14.1 \mathrm{~m}$ from the source. The surface of the mirrors was polished to a rms roughness less than $3 \AA$ and to a rms longitudinal slope error less than $2 \mu$ rad. The first mirror is indirectly water cooled through two Ni plated $\mathrm{Cu}$ blades immersed in In-Ga liquid eutectic in two grooves in the mirror surface. 
The collimation of the beam is ensured by the first mirror and allows the experimenter to overcome the natural divergence of the beam from the bending magnet source, this improves the energy resolution without limiting the vertical aperture of the primary slits. The vertical aperture is then ranged between 0.08 and $0.55 \mathrm{mrad}$ depending on the beamline working energy. The best collimation is obtained by adjusting the mirror bending radius for each grazing incidence angle of the mirror in order to reduce the Full Width Half Maximum (FWHM) of a characteristic sharp structure of a well known sample. This is for example illustrated in Figure 2 with the measurements of the XANES spectrum of $\mathrm{KMnO}_{4}$ at $6.5 \mathrm{mrad}$ of vertical grazing incidence. Depending on the horizontal acceptance of the beamline, different bending radii are found and must be adjusted accordingly for providing the best resolution. The measured FWHM of the pre-edge structure of $\mathrm{KMnO}_{4}$ at the $\mathrm{Mn} \mathrm{K}$ edge $(6540 \mathrm{eV})$ is $1.53 \mathrm{eV}$ and that of the pre-edge structure of (TBA) $\mathrm{Mo}_{2} \mathrm{O}_{7}$ at the $\mathrm{Mo} \mathrm{K}$ edge $(20000 \mathrm{eV})$ is $4.80 \mathrm{eV}$, both obtained with a $15 \mathrm{~mm}$ horizontal aperture $(1.17 \mathrm{mrad})$. These values must be compared to the quadratic sum of the intrinsic Darwin resolution of the used $\mathrm{Si}(220)$ monochromator [6] $(0.38 \mathrm{eV}$ at $6.5 \mathrm{keV}$ and $1.16 \mathrm{eV}$ at $20 \mathrm{keV}$ ) and to the $\mathrm{K}$ edge core-hole width of the absorbing atom [7] (1.16 eV for Mn and $4.52 \mathrm{eV}$ for Mo). Taking into account these parameters, the theoretical overall energy resolution of the beamline is $1.22 \mathrm{eV}$ and $4.67 \mathrm{eV}$ for $\mathrm{Mn}$ and Mo $\mathrm{K}$ edges, respectively. Experimental and theoretical values are closely related emphasizing the good performances of SAMBA in term of energy resolution. The bending radius of the second mirror is adjusted for providing at the sample position a focusing beam. The optimal vertical size is $110 \mu \mathrm{m}$ at the FWHM with a foot size of about $300 \mu \mathrm{m}$.
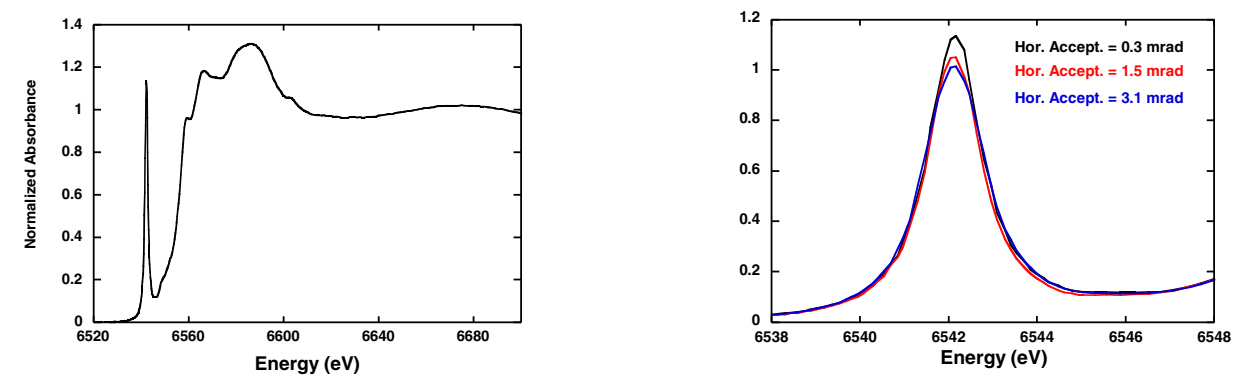

Figure 2. Optimisation of the collimation provided by the first mirror tilted at $6.5 \mathrm{mrad}$ for different horizontal acceptances defined by the primary slits. The optimisation is made by changing the bending radii of the mirror and looking at the FWHM of the pre-edge structure observed at $6542 \mathrm{eV}$ for $\mathrm{KMnO}_{4}$.

\subsection{Sagittally focusing double crystal monochromator}

The sagittally focusing Double Crystal Monochromator (DCM) provided by Oxford Danfysik is used in the so-called high flux mode. The DCM is installed at $16.1 \mathrm{~m}$ from the source. It deals of a first flat water-cooled $\mathrm{Si}(220)$ crystal and a sagittally bent $2^{\text {nd }}$ crystal. The main Bragg axis of rotation passes through the centre of the diffracting face on the $1^{\text {st }}$ crystal and perpendicular to the beam axis. In this way, the incident white beam is always centred on the axis of rotation. The desired energy is selected, according the Bragg reflection, by rotating the crystal, and then changing the Bragg angle. The EXAFS spectra are recorded by changing step by step the Bragg angle in order to cover about $1000 \mathrm{eV}$ above the edge of interest. A fixed offset to the exit beam is achieved by moving the $2^{\text {nd }}$ crystal perpendicularly to the first one according to the Bragg angle. In this case the beam moves along the axis of the sagittallybent $2^{\text {nd }}$ crystal. The first crystal is indirectly water-cooled by using a multi channel water cooling support (SOLEIL original design) in contact with the crystal through a thin film of In-Ga eutectic. The $2^{\text {nd }}$ crystal is uncooled. The resultant difference in lattice spacing between the $1^{\text {st }}$ and $2^{\text {nd }}$ crystals due to this temperature effect is compensated by a small deviation from parallelism of the second crystal, thus the outgoing beam exits marginally off-parallel. The horizontal acceptance of the monochromator 
is not limited by the size of the $1^{\text {st }}$ crystal but by the efficiency of the sagittal bender. The shape of the beam for an horizontal acceptance of $1.5 \mathrm{mrad}$ (corresponding to $25 \mathrm{~mm}$ of horizontal primary slits) is displayed Figure 3(a). For larger horizontal acceptance $(\sim 3.7 \mathrm{mrad})$, although the flux of photons at the sample position increases, the shape of the spot is larger and horizontally prolonged by two long wings that could be cleaned only by slits very close to the focus point (Figure 3(b)).

In the high flux mode, the bender follows a repeatable law as a function of the photon energy for keeping constant the size of the beam at the sample position during an EXAFS scan. The usual beam size available in this so-called dynamically sagittal focusing mode is around $300 \mu \mathrm{m}(\mathrm{H}) \times 200 \mu \mathrm{m}(\mathrm{V})$ (FWHM). Nevertheless, in order to avoid possible radiation damage on the sample due to the high density of photons in a so small spot, the beamline is often slightly defocused in both directions. In this case, the size of the beam can be as large as $2 \mathrm{~mm}(\mathrm{H}) \times 1 \mathrm{~mm}(\mathrm{~V})$.

(a)

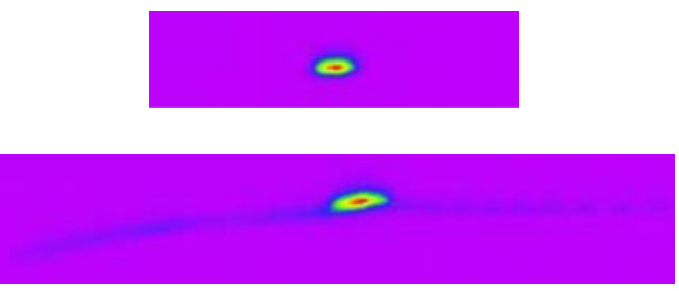

Figure 3. Shape of the sagittally focused beam at the sample position at $8.5 \mathrm{keV}$ for (a) 1.5 and (b) $3.7 \mathrm{mrad}$ of horizontal acceptance ( $\mathrm{Si}(220) \mathrm{DCM}, 400 \mathrm{~mA}$ stored current and $6.5 \mathrm{mrad}$ vertical acceptance).

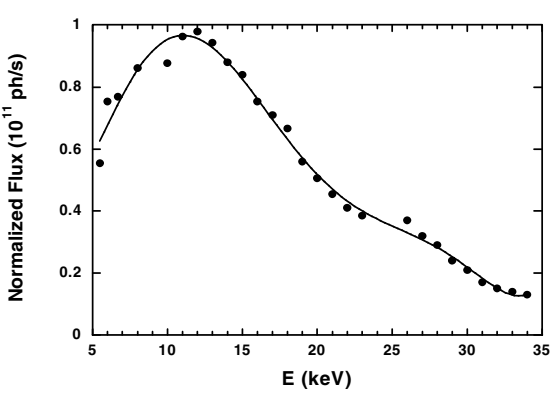

Figure 4. Normalized flux at the sample position for the $\mathrm{Si}(220)$ sagittally DCM with respect to the vertical acceptance $(400 \mathrm{~mA}$ stored current, $1.5 \mathrm{mrad}$ horizontal and $1 \mathrm{mrad}$ vertical acceptance).

Figure 4 displays the available flux at the sample position provided by the commonly installed $\mathrm{Si}(220)$ sagittally DCM. This curve obtained for an horizontal acceptance of $1.5 \mathrm{mrad}$, is normalized by the vertical acceptance on the mirrors. Then a typical flux at the sample position at $8.5 \mathrm{keV}$ is $510^{11} \mathrm{ph} / \mathrm{s}$ (6 mrad of vertical acceptance and $1.5 \mathrm{mrad}$ of horizontal acceptance). Nevertheless, in experiments requiring more flux of photons we can change the $\mathrm{Si}(220)$ crystals by $\mathrm{Si}(111)$ crystals. In this case the energy range available is limited from 4 to $20 \mathrm{keV}$.

Both crystals can be vertically translated in opposite directions when we want to provide pink beam on the second Quick-EXAFS monochromator.

\subsection{Quick-EXAFS monochromator}

The SOLEIL Quick-EXAFS monochromator is an up-graded version of the monochromator recently developed by the Frahm's group [8] which deals with a channel cut crystal mounted on a cam driven tilt table that oscillates around a selected central Bragg angle, $\theta$, with a user selectable angular amplitude, $\Delta \theta(\mathrm{t})$ ranging from 0.1 to $1.5^{\circ}$. The angular amplitude is defined by a mechanical eccentric disk directly mounted inside the monochromator. Changing the amplitude requires the opening of the monochromator to replace the eccentric disk. The monochromator installed on SAMBA, shown in Figure 5, is based on the same principle.

Most important improvements made by the R \& D SOLEIL division on the SAMBA Quick-EXAFS monochromator are i) its wide Bragg angle range up to $4^{\circ}$ allowing the recording of EXAFS data with spectral range up to $1000 \mathrm{eV}$ or more at any edge covered by the beamline (from the Ti to the Cs $\mathrm{K}$ edges), ii) the remote change of the Bragg oscillation amplitude through an original high vacuum variable cam design iii) the remote change of the monochromator crystals from $\mathrm{Si}(111)$ to $\mathrm{Si}(311)$ 


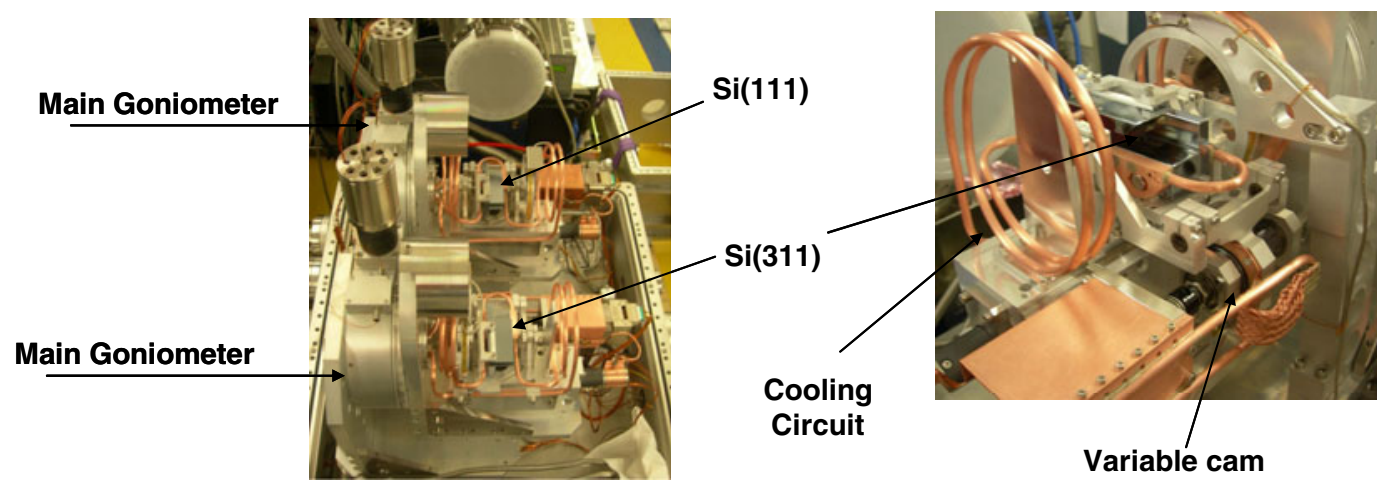

Figure 5. Quick-EXAFS monochromator installed on the SAMBA beamline.

allowing a permanent access to the $4-36 \mathrm{keV}$ energy range. The improvements made on the SAMBA Quick-EXAFS monochromator avoid a long-time maintenance procedure (for changing amplitude oscillation of crystals or energy range) and definitely increase beamline performances offering new experimental possibilities. For instance, the XAS study of the same bimetallic catalyst at both edges during the catalytic reaction is now possible with the SAMBA's Quick-EXAFS set-up, even if the energy of the two edges is so different that two distinct crystals are required.

Presently the best performance of the Quick-EXAFS set-up in terms of oscillation frequency is $40 \mathrm{~Hz}$. Actually recording speed could be double since two spectra are recorded per cycle: one while increasing the Bragg angle and one on the way back. But practically, due to limitation on ionization chambers and electronics used for the detection of the signal, both directions do not produce identical spectra above $1 \mathrm{~Hz}$ and the fastest recommended acquisition rate in transmission detection mode is $8 \mathrm{~Hz}$ which guarantees that the acquired signal is comparable with the signal recorded with the step by step Oxford sagittally focusing DCM. Figure 6 gives an example of comparison of Quick-EXAFS spectra recorded at different oscillation frequencies with the EXAFS data recorded for the same sample with the Oxford DCM.

Transmission and fluorescence experiments are both available in this time-resolved mode of acquisition using the SAMBA's Quick-EXAFS monochromator. The first tests done in fluorescence, using an avalanche photodiode as detector, indicate a detection limit around $10 \mathrm{mmol}$ for $\mathrm{Cu}^{2+}$ solution with typical oscillation frequencies ranging from 0.1 to $0.01 \mathrm{~Hz}$ for a whole EXAFS spectrum.

(a)

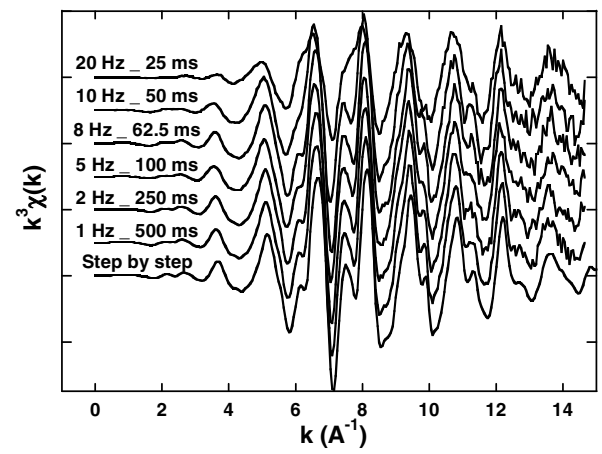

(b)

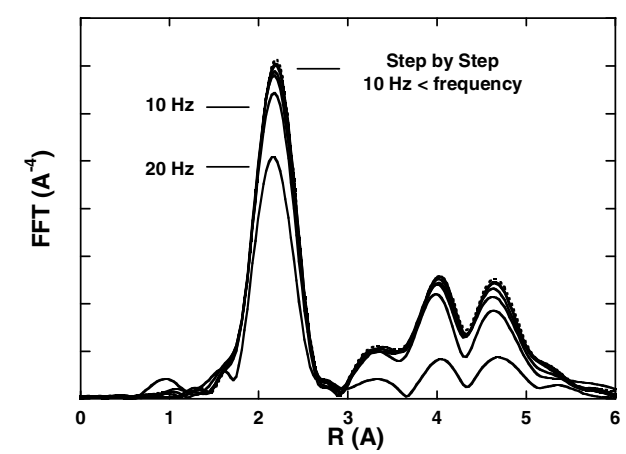

Figure 6. Comparison of Quick-EXAFS spectra recorded for a Ni metallic foil at different oscillation frequencies with the step by step EXAFS spectrum recorded for the same sample with the Oxford DCM. (a) EXAFS spectra and (b) corresponding Fourier Transforms. 
The limitation of the acquisition to the XANES energy range allows for improving the time resolution by factor 10, with data recorded in $500 \mathrm{~ms}$.

\subsection{XAS experimental hutch}

The first experimental hutch is used for conventional XAS experiments, working in both operation modes for the beamline: i.e the high flux mode or the time-resolved mode. As presented in Figure 7, the station is equipped with a granite table supporting a long rail aligned along the beam direction on which are mounted an X-Z-R $\mathrm{R}_{Z}$ remotely controlled sample holder at $30 \mathrm{~m}$ from the source. Three Oxford or Ohken ionization chambers are used for transmission detection, whereas different fluorescence detectors can be mounted at $90^{\circ}$ from the incident beam for fluorescence detection. The beamline is equipped with a Vortex Silicon Drift detector with $50 \mathrm{~mm}^{2}$ of active area and a 7-elements Ge solid state detector. A future development in fluorescence detection will be obtained with the purchase of a new multi-element detector with greater performances than the 7-elements Ge detector.

This station is characterized by a number of modular sample environments including thermostated liquid cells, liquid $\mathrm{N}_{2}$ and He cryostats, ovens for heating samples up to $600{ }^{\circ} \mathrm{C}$ under controlled atmosphere with a distribution rack for gases and a mass spectrometer for study of reaction products. All these cells are suitable for both transmission and fluorescence detection modes. A home-made goniometric set-up is used for the measurements of thin films in grazing incidence. The beamline is also equipped with complementary techniques. Raman and UV-Vis spectroscopies and Differential Scanning Calorimetry are then often simultaneously combined with the XAS time-resolved measurements for providing complementary information during dynamical processes allowing a better understanding of the system reactivity [9-11].

(a)

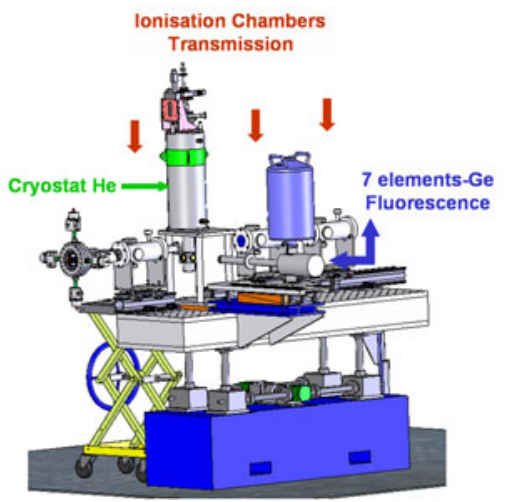

(b)

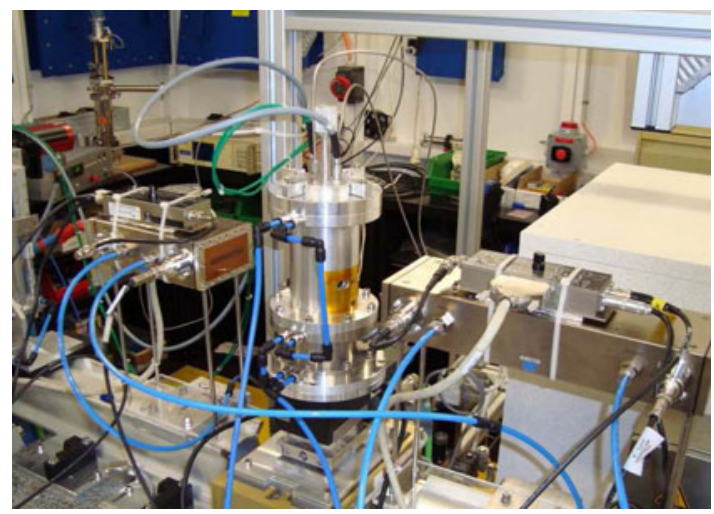

Figure 7. (a) XAS station with the granite table, the ionization chambers for transmission and the 7-elements Ge detector for fluorescence. At the sample position, a liquid He cryostat is positioned for the study of samples at low temperatures. (b) View of the oven used for Operando study of catalysts.

\subsection{SurfAS experimental hutch}

The Surface Absorption Spectroscopy (SurfAS) hutch is dedicated to the study of thin films and surfaces. Since it is not possible to protect against atmosphere films below a few monolayers or most surfaces, it is necessary to clean and prepare them in-situ right before measurements. The UHV system is an upgrade of that transferred from beamline SEXAFS (DW21-LURE). Up to three thermal or electron beam evaporators can be installed when provided by users, while one thermal and one electron beam evaporator from Omicron Gmbh are available upon request. An Auger CMA100 unit, a LEED apparatus, a Prevac 0.15-5 keV sputtering gun, a 7 elements Canberra fluorescence detector and a total electron 
yield detector complete the environment. Sample can be cooled to liquid nitrogen or heated up to $950{ }^{\circ} \mathrm{C}$ with the standard UHV manipulator capable of $\mathrm{X}, \mathrm{Y}, \mathrm{Z}$ and $\mathrm{R}_{Z}$ movements. A gas distribution system allows the user to introduce low doses of high purity gases for surface reactions or Ar sputtering. Four samples can be introduced in a first UHV chamber through a fast load lock, before being studied in the main vessel.

The hutch is the last one of the beamline allowing users to familiarize with the apparatus while others may work undisturbed in the general purpose EXAFS hutch, maximizing beamline efficiency. Detectors of the EXAFS hutch are replaced by a pipe $(0.1 \mathrm{mbar})$ and the hutch is no more accessible, when transferring the beam to the SurfAS hutch.

\section{References}

[1] Teo B.K., EXAFS: Basic Principles and Data Analysis (Springer, New York, 1986).

[2] Koningsberger D.C. and Prins R., (Eds), X-ray Absorption: Principles, Applications, Techniques of EXAFS, SEXAFS and XANES (Wiley, New York, 1988).

[3] Bunker G., Introduction to XAFS: A Practical Guide to X-ray Absorption Fine Structure Spectroscopy (Cambridge University Press, 2010).

[4] Ankudinov A.L., Ravel B., Rehr J.J. and Conradson S.D. Phys. Rev. B 58 (1998) 7565-7576.

[5] Belin S., Briois V., Traverse A., Idir M., Moreno T. and Ribbens M., Physica Scripta T115 (2005), 980-983.

[6] http://www.chess.cornell.edu/oldchess/operatns/xrclcdwn.htm.

[7] Krause M.O. and Oliver J. H., J. Phys. Chem. Ref. Data 8 (1979) 329-338.

[8] Frahm, R.M. Richwin and D. Lützenkirchen-Hecht, Physica Scripta T115 (2005) 974-976.

[9] La Fontaine C., Villain F., Briois V., Sécordel X., Yoboué A., Berrier E. and Cristol S., I. Harrick Scientific Products (Ed.), Applications Note n91201 (2010).

[10] Stötzel J., Lützenkirchen-Hecht D., Frahm R., Santilli C.V., Pulcinelli S.H., Kaminski R., Fonda E., Villain F. and Briois V., J. Phys. Chem. C 114 (2010) 6228-6236.

[11] Vantelon D., Lagarde P., Flank A.-M., Berrier E., Secordel X., Cristol S., La Fontaine C., Villain F. and Briois V., Phase Transitions 82 (2009) 322-335. 